\title{
Development of an efficient conjugation-based genetic manipulation system for Pseudoalteromonas
}

\author{
Pengxia Wang ${ }^{1}$, Zichao Y ${ }^{2}$, Baiyuan $\mathrm{Li}^{1,3}$, Xingsheng Cai ${ }^{1}$, Zhenshun Zeng ${ }^{1,3},{\text { Xiulan } \text { Chen }^{2} \text { and Xiaoxue Wang }}^{\text {1* }^{*}}$
}

\begin{abstract}
Pseudoalteromonas is commonly found throughout the world's oceans, and has gained increased attention due to the ecological and biological significance. Although over fifty Pseudoalteromonas genomes have been sequenced with an aim to explore the adaptive strategies in different habitats, in vivo studies are hampered by the lack of effective genetic manipulation systems for most strains in this genus. Here, nine Pseudoalteromonas strains isolated from different habitats were selected and used as representative strains to develop a universal genetic manipulation system. Erythromycin and chloramphenicol resistance were chosen as selection markers based on antibiotics resistance test of the nine strains. A conjugation protocol based on the RP4 conjugative machinery in E. coli WM3064 was developed to overcome current limitations of genetic manipulation in Pseudoalteromonas. Two mobilizable gene expression shuttle vectors (pWD2-oriT and pWD2Ery-oriT) were constructed, and conjugation efficiency of pWD2-oriT from E. coli to the nine Pseudoalteromonas strains ranged from $10^{-6}$ to $10^{-3}$ transconjugants per recipient cells. Two suicide vectors, pK18mobsacB-Cm and pK18mobsacB-Ery (with sacB for counter-selection), were constructed for gene knockout. To verify the feasibility of this system, we selected gene or operon that may lead to phenotypic change once disrupted as targets to facilitate in vivo functional confirmation. Successful deletions of two genes related to prodigiosin biosynthesis (pigMK) in P. rubra DSM 6842, one biofilm related gene (bsmA) in P. sp. SM9913, one gene related to melanin hyperproduction (hmgA) in P. lipolytica SCSIO 04301 and two flagella-related genes (fliF and fliG) in P. sp. SCSIO 11900 were verified, respectively. In addition, complementation of hmgA using shuttle vector pWD2-oriT was rescued the phenotype caused by deletion of chromosomal copy of hmgA in P. lipolytica SCSIO 04301. Taken together, we demonstrate that the vectors and the conjugative protocol developed here have potential to use in various Pseudoalteromonas strains.
\end{abstract}

Keywords: Pseudoalteromonas, Conjugation, Genetic manipulation, Marine bacteria

\section{Background}

Genus Pseudoalteromonas belongs to the Gammaproteobacteria class with thirty-eight recognized species reported so far [1,2]. Pseudoalteromonas is ubiquitous in the marine environment [2-10], and many strains have been isolated from deep sea [8], polar sea $[2,9,10]$, or other extreme marine habitats, highlighting their important and diverse role in marine ecosystems. Pseudoalteromonas strains also produce a range of bioactive compounds with

\footnotetext{
* Correspondence: xxwang@scsio.ac.cn

'Key Laboratory of Tropical Marine Bio-resources and Ecology, Guangdong Key Laboratory of Marine Materia Medica, RNAM Center for Marine Microbiology, the South China Sea Institute of Oceanology, Chinese Academy of Sciences, Guangzhou 510301, China

Full list of author information is available at the end of the article
}

antimicrobial, antifouling, or algicidal activities that have attracted global attentions from microbiologist, ecologists and chemists [11]. To date, over fifty Pseudoalteromonas genomes have been sequenced, laying a solid foundation for comparative studies on their adaptability to ecological niches as well as for the discovery of novel natural products. Several studies have used ectopic expressing genes in E. coli as a complementary means to interrogate genes and their functions in Pseudoalteromonas [12,13]. However, the lack of an efficient and universal genetic manipulation system has limited the comparative studies of Pseudoalteromonas at the molecular level in vivo.

Shuttle vector pWD2 has been successfully isolated previously and can be used as an expression vector in its 
original derived strain P. sp. SM20429 [9]. Direct transfer of non-mobilizable pWD2 to other Pseudoalteromonas strains is constrained by the need for electroporation. Electroporation does not seem to work in majority of Pseudoalteromonas strains whose growth are usually saltdependent. Based on our current knowledge, to date, gene deletion systems have only been described for two Pseudoalteromonas strains, $P$. haloplanktis TAC125 and $P$. sp. SM9913 [14,15]. Both protocols were designed for the construction of strain-specific isogenic knockouts, thus developing a widely applicable genetic manipulation system for Pseudoalteromonas now becomes a priority.

A few common features of Pseudoalteromonas make genetic manipulation difficult. Harboring multidrug resistance genes and multiple drug efflux pumps in the genome $[8,16]$ can equip cells to survive antibiotic pressure and also can develop further mutations in genes encoding the target sites of antibiotics [17]. Abundant distribution of restriction-modification systems also reduces transformation efficiency by degrading foreign DNAs [18]. In addition, commonly used conjugation protocol does not offer a condition that allow decent growth of the nonmarine originated mesophilic donor strain and the marine recipient Pseudoalteromonas strains. Solving these problems is critical for developing efficient genetic manipulation systems for Pseudoalteromonas.

Bacterial conjugation is a genetic exchange mechanism that requires direct contact between donor and recipient cells. Bacterial conjugation machinery is composed of an oriT sequence and tra genes [19]. The oriT sequence needs to be provided by the plasmid in cis, while the tra genes, which encode a relaxase, a mating pair formation complex, and a type IV coupling protein, can be provided in cis or in trans. The relaxase cleaves the nic site within the oriT sequence and covalently attaches to the $5^{\prime}$ end of the transferred strand to produce a single-strand DNA (ssDNA)-relaxase complex with other auxiliary proteins; this is termed the relaxosome [19]. The type IV coupling protein mediates the connection between the relaxasome and the mating pair formation complex, the latter being the secretion system that transfers ssDNA-relaxase complex into recipient cells [20]. Since the DNA transferred by conjugation is single-stranded instead of double-stranded transferred by electroporation, thus it could reduce the possible degradation by restrictionmodification systems which preferably degrading doublestranded DNAs [18]. As a result, conjugation techniques have been widely used for genetic manipulations in Gramnegative bacteria and have also been reported in several Gram-positive bacteria (reviewed in [21]).

Here, we present an efficient conjugation-based genetic manipulation system for Pseudoalteromonas. Nine Pseudoalteromonas strains from different habitats were selected to represent strains from deep-sea sediment, Arctic sea ice, deep-sea hydrothermal vent, Mediterranean coastal water, Antarctic surface seawater, and sediment or surface water in the South China Sea. Based on antibiotic sensitivity test, two different resistance genes are used for selection to construct new vectors for gene expression and gene knockout. A conjugal transfer system with a modified medium using these vectors is developed, and feasibility of this transferring system is confirmed in nine Pseudoalteromonas strains. We further demonstrate that targeted deletion mutants are successfully constructed in four Pseudoalteromonas strains using this system to facilitate studies of these genes or operons in vivo, including P. rubra DSM 6842, P. sp. SM9913, P. lipolytica SCSIO 04301 and P. sp. SCSIO 11900. In addition, gene complementation using this system is also confirmed in one deletion mutant of $P$. lipolytica SCSIO 04301.

\section{Results and discussion}

Antibiotic resistance in different Pseudoalteromonas strains To develop a universal genetic manipulation system for a variety of Pseudoalteromonas strains, nine Pseudoalteromonas strains were selected as representative strains (Table 1). The ability to resist different antibiotics of each strain was first analyzed in order to find the most suitable resistance markers for maintaining the vectors in proper host (Additional file 1: Table S1). All nine Pseudoalteromonas strains were sensitive to $25 \mu \mathrm{g} / \mathrm{ml}$ erythromycin and $30 \mu \mathrm{g} / \mathrm{ml}$ chloramphenicol. None of the nine strains was sensitive to gentamicin, kanamycin, spectinomycin, and tetracycline. Resistance to apramycin and ampicillin is partial in some strains thus not suitable for constructing universal vectors. Taken together, erythromycin and chloramphenicol resistance genes serve as good candidates of constructing vectors for universal gene expression and gene knockout in Pseudoalteromonas.

\section{Construction of mobilizable shuttle vectors for Pseudoalteromonas}

Initially we made many attempts to electroplate vector pWD2 [9] to SM9913 and A37-1-2. Transformation efficiencies remained extremely low, despite that electroporation of pWD2 into its original host strain was successful using a similar, previously described method [9]. As noted above, electroporation is of only very limited use in Pseudoalteromonas, warranting the use of a conjugationapproach. To conduct conjugation, the pWD2-oriT mobi lizable plasmid (Figure 1A) was constructed by inserting the mobilization module from pBBR1MCS-2 [22] into the E. coli-Pseudoalteromonas shuttle vector pWD2 [9]. The mobilizable module, which contains the mob gene and oriT sequence, was amplified using the BamHI site-flanking primer set oriT-F/oriT-R (Additional file 1: Table S2) and ligated into the BamHI sites of pWD2. Vector pWD2-oriT therefore acts as a shuttle vector that can be mobilized by 
Table 1 Bacterial strains and plasmids used in this study

\begin{tabular}{|c|c|c|}
\hline Strains/plasmids & Description $^{\mathrm{a}}$ & Reference \\
\hline \multicolumn{3}{|c|}{ Pseudoalteromonas strains } \\
\hline SM9913 & P. sp. SM9913, deep-sea sediment at a water depth of 1855 meters near the Okinawa Trough, $20^{\circ} \mathrm{C}$ & [8] \\
\hline A37-1-2 & P. arctica A37-1-2, Arctic sea ice strain, $20^{\circ} \mathrm{C}$ & [7] \\
\hline DSM 16099 & P. spiralis DSM 16099, deep ocean hydrothermal vents of the Juan de Fuca Ridge, $30^{\circ} \mathrm{C}$ & [35] \\
\hline DSM 16098 & P. telluritireducens DSM 16098 , deep ocean hydrothermal vents of the Juan de Fuca Ridge, $30^{\circ} \mathrm{C}$ & [35] \\
\hline DSM 6842 & P. rubra DSM 6842 , Mediterranean coastal waters off Nice, $25^{\circ} \mathrm{C}$ & {$[26,36]$} \\
\hline SM20429 & P. sp. SM20429, plasmid curing mutant of P. sp. BSi20429, Arctic sea ice strain, $20^{\circ} \mathrm{C}$ & [9] \\
\hline TAC125 & P. haloplanktis TAC125, Antarctic surface seawater, $20^{\circ} \mathrm{C}$ & [37] \\
\hline SCSIO 04301 & P. lipolytica SCSIO 04301, sediment at $63 \mathrm{~m}$ deep in the South China Sea $\left(18^{\circ} \mathrm{O}=\mathrm{N}, 109^{\circ} 42=\mathrm{E}\right), 25^{\circ} \mathrm{C}$ & [28] \\
\hline SCSIO 11900 & P. sp. SCSIO 11900 , surface mucus layer of the coral at $4 \mathrm{~m}$ deep in the South China Sea $\left(18^{\circ} 13=\mathrm{N}, 109^{\circ} 28=\mathrm{E}\right), 25^{\circ} \mathrm{C}$ & [28] \\
\hline$\triangle b s m A$ & bsmA gene deletion mutant of P.sp. SM9913 & This study \\
\hline$\Delta p i g M-K$ & Deletion mutant of the DNA region containing pigM-K genes related to prodigiosin biosynthesis in $P$. rubra DSM 6842 & This study \\
\hline$\Delta h m g A$ & hmgA gene deletion mutant of $P$. lipolytica SCSIO 04301 & This study \\
\hline$\Delta$ fliFG & Deletion mutant of the DNA region containing fliFG genes encoding flagellar motor proteins in P. sp. SCSIO 11900 & This study \\
\hline \multicolumn{3}{|c|}{ Escherichia. coli strain } \\
\hline WM3064 & RP4 (tra) in chromosome, DAP', $37^{\circ} \mathrm{C}$ & [23] \\
\hline \multicolumn{3}{|l|}{ Plasmids } \\
\hline pWD2 & E. coli and Pseudoalteromonas shuttle vector, $\mathrm{Amp}^{r}, \mathrm{Cm}^{r}$ & [9] \\
\hline pHT304 & E. coli and Bacillus thuringiensis shuttle vector, Ampr, Eryr thuringiensis shuttle vector, Ampr', Ery ${ }^{r}$ & [30] \\
\hline pBBR1MCS-2 & Broad-host-range cloning vector, Kan ${ }^{r}$ & [22] \\
\hline pWD2-oriT & pWD2 containing $1.5 \mathrm{~kb} B a m H I$ fragment with mobilization region from pBBR1MCS-2, Ampr, $\mathrm{Cm}^{r}$ & This study \\
\hline pWD2Ery-oriT & pWD2-oriT containing a 900bp erythromycin resistant gene replaced the chloramphenicol resistant gene, Ampr', Ery ${ }^{r}$ & This study \\
\hline pK18mobsacB & Widely used gene knockout vector, Kan ${ }^{r}$ & [24] \\
\hline pK18mobsacB-Cm & pK18mobsacB containing the chloramphenicol resistant gene from pWD2, $\mathrm{Kan}^{r}, \mathrm{Cm}^{r}$ & This study \\
\hline pK18mobsacB-Ery & pK18mobsacB containing the erythromycin resistant gene from pHT304, Kan', Ery ${ }^{r}$ & This study \\
\hline pK18Cm-bsmA & pK18mobsacB-Cm containing the homologous arms of bsmA gene of SM9913, $\mathrm{Kan}^{r}, \mathrm{Cm}^{r}$ & This study \\
\hline pK18Ery-pigM-K & pK18mobsacB-Ery containing the homologous arms of the pigM-pigK DNA region of DSM 6842, Kan', Ery ${ }^{r}$ & This study \\
\hline pK18Ery-hmgA & pK18mobsacB-Ery containing the homologous arms of hmgA gene of SCSIO 04301, Kan'r, Ery ${ }^{r}$ & This study \\
\hline pK18Ery-fliFG & pK18mobsacB-Ery containing the homologous arms of the fliF-fliG DNA region of SCSIO 11900, Kann', Ery' & This study \\
\hline
\end{tabular}

${ }^{a} A m p^{r}$, ampicillin resistance; Ery ${ }^{r}$, erythromycin resistance; Kan ${ }^{r}$, kanamycin resistance; $\mathrm{Cm}^{r}$, chloramphenicol resistance.

Temperature indicates the optimal growth temperature of each strain.

the RP4 conjugative machinery in trans between E. coli and Pseudoalteromonas. To offer an alternative selection marker, the pWD2Ery-oriT vector (Figure 1B) was also constructed by replacing the chloramphenicol resistance gene with the erythromycin resistance gene at the same site.

\section{Conjugation of pWD2-oriT between $E$. coli and Pseudoalteromonas}

The widely used conjugation donor strain E. coli WM3064 has RP4 Tra function integrated in the chromosome, and is an auxotrophic strain whose growth relies on the supplementation of diaminopimelic acid (DAP) in the medium [23]. First, the pWD2-oriT shuttle vector was transformed into E. coli WM3064 to obtain E. coli WM3064/pWD2oriT. Using it as a donor strain, pWD2-oriT was then transferred into nine recipient Pseudoalteromonas strains individually by intergeneric conjugation. The conjugation protocol was optimized to allow decent simultaneous growth of the donor and recipient strains. Different conjugation temperatures $\left(15,25,30\right.$, or $\left.37^{\circ} \mathrm{C}\right)$ and media with different salt concentrations (LB+DAP, modified LB+DAP, or 2216E+DAP) were tested, respectively. Since E. coli WM3064 is able to grow at temperatures as low as $15^{\circ} \mathrm{C}$, the optimal mating temperature was thus determined by the optimal growth temperature of the recipient strain (shown in Table 1). Growth of E. coli WM3064 and Pseudoalteromonas was also affected by the salt concentration in the medium. E. coli WM3064 grew very slowly in 2216E+DAP medium which has high salt concentration, and most Pseudoalteromonas strains had poor growth in 

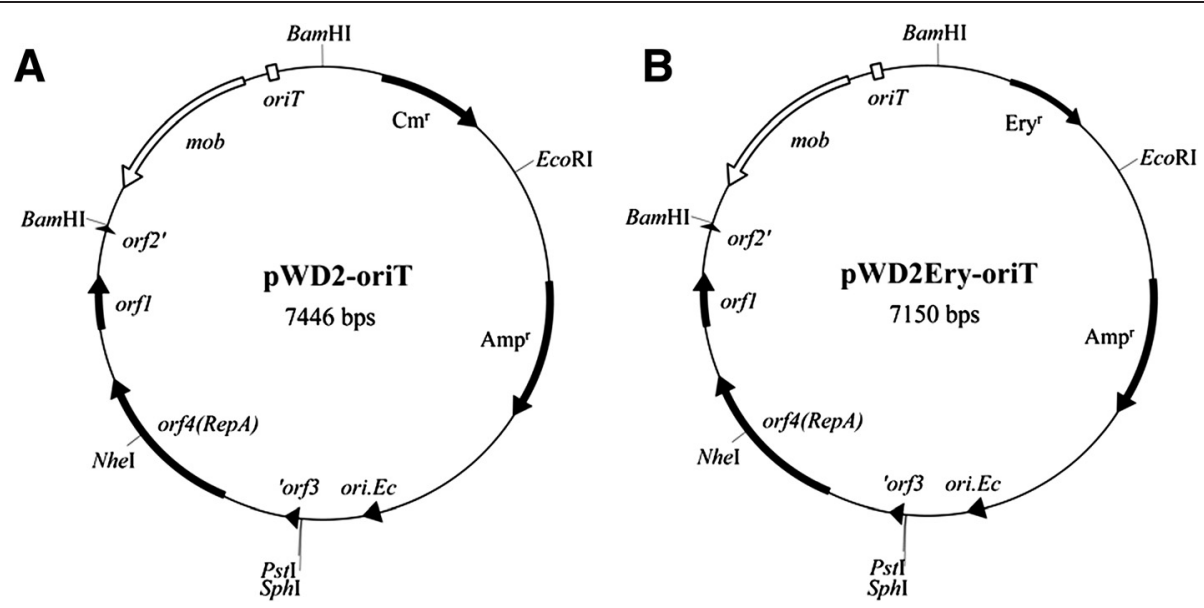

Figure 1 Maps of the pWD2-oriT (A) and pWD2Ery-oriT (B) mobilizable shuttle vectors.

LB medium (Additional file 1: Figure S1). Since viability of the donor strain during mating appeared to be a more important determining factor for conjugation efficiency, modified LB with moderate salt concentration was chosen for E. coli WM3064-Pseudoalteromonas mating to guarantee growth of both donor and recipient strains (Additional file 1: Figure S1).

To evaluate the conjugation protocol, approximately $10^{6}$ mid-exponential phase E. coli and Pseudoalteromonas cells were washed twice with antibiotic-free mating medium (modified LB) and used for each conjugation. Chloram phenicol-resistant transconjugants were counted to calculate the transfer efficiency of pWD2-oriT. Colony PCR using pWD2-oriT-specific primers was used to confirm the presence of the transferred plasmid by isolating plasmids from randomly selected transconjugants. In addition, strain-specific RAPD-PCR was used to characterize and identify the resulting recipient strains to ensure no false positive colonies obtained. Conjugation was successful for all nine strains (Table 2, Additional file 1: Figure S2). At least twelve colonies from each recipient strain were randomly screened, and no false positive colonies were detected. Transfer efficiencies varied from $10^{-6}$ to $10^{-3}$ transconjugants per recipient cells (Table 2). Collectively, these results suggest that the mobilizable shuttle vector pWD2-oriT can be transferred to various Pseudoalteromonas strains by conjugation.

\section{Construction of gene knockout vectors for Pseudoalteromonas}

As shown in Figure 2, two derivatives vectors (pK18mob$s a c B-\mathrm{Cm}$ and pK18mobsacB-Ery) were constructed by inserting chloramphenicol or erythromycin resistance genes into the BamHI/EcoRI site of the gene knockout vector pK18mobsacB [24]. The vectors both contain the broad-host-range transfer machinery of plasmid RP4 and a modified sacB gene from Bacillus subtilis [24]. Gene $s a c B$ encodes levansucrase, which catalyzes the hydrolysis of sucrose and synthesizes levans. Levans are high molecular weight fructose polymers that are fatal to most Gram-negative bacteria [25]; $s a c B$ can therefore be used as a counter-selectable marker in these Gram-negative bacteria. The modified pK18mobsacB vector is suicidal in Pseudoalteromonas as its host range is restricted to E. coli and closely related species such as Salmonella and Serratia; therefore it can be used for gene knockout in Pseudoalteromonas. To verify the feasibility of this system, four Pseudoalteromonas strains, DSM 6842, SM9913, SCSIO 04301 and SCSIO 11900, covering high or low conjugation efficiencies for pWD2-oriT transfer, were used as recipient strains. To facilitate the verification of deletion mutants, we specifically selected gene or operon that may lead to phenotypic changes once disrupted as targets for functional confirmation.

Table 2 Transfer efficiencies of pWD2-oriT between E. coli and Pseudoalteromonas strains

\begin{tabular}{llll}
\hline Recipient strains & CFU* & Transconjugants $^{\&}$ & Efficiency \\
\hline A37-1-2 & $4.7 \times 10^{7}$ & $3.0 \times 10^{4}$ & $6.4 \times 10^{-4}$ \\
TAC125 & $2.5 \times 10^{7}$ & $1.8 \times 10^{2}$ & $7.2 \times 10^{-6}$ \\
SM20429 & $3.2 \times 10^{7}$ & $1.4 \times 10^{4}$ & $4.4 \times 10^{-4}$ \\
SM9913 & $3.8 \times 10^{7}$ & $1.6 \times 10^{4}$ & $4.2 \times 10^{-4}$ \\
DSM 16099 & $1.5 \times 10^{8}$ & $1.6 \times 10^{3}$ & $1.1 \times 10^{-5}$ \\
DSM 16098 & $9.5 \times 10^{7}$ & $1.3 \times 10^{2}$ & $1.4 \times 10^{-6}$ \\
DSM 6842 & $8.7 \times 10^{7}$ & $9.4 \times 10^{1}$ & $1.1 \times 10^{-6}$ \\
SCSIO 04301 & $9.7 \times 10^{7}$ & $1.6 \times 10^{5}$ & $1.6 \times 10^{-3}$ \\
SCSIO 11900 & $3.0 \times 10^{7}$ & $9.8 \times 10^{1}$ & $3.3 \times 10^{-6}$
\end{tabular}

${ }^{*}$ Average number of recipient cells in two replicates.

${ }^{\&}$ Average number of transconjugants in two replicate conjugations plated in triplicate plates; approximately $1 / 10^{\text {th }}$ of the total conjugation volume was plated. 

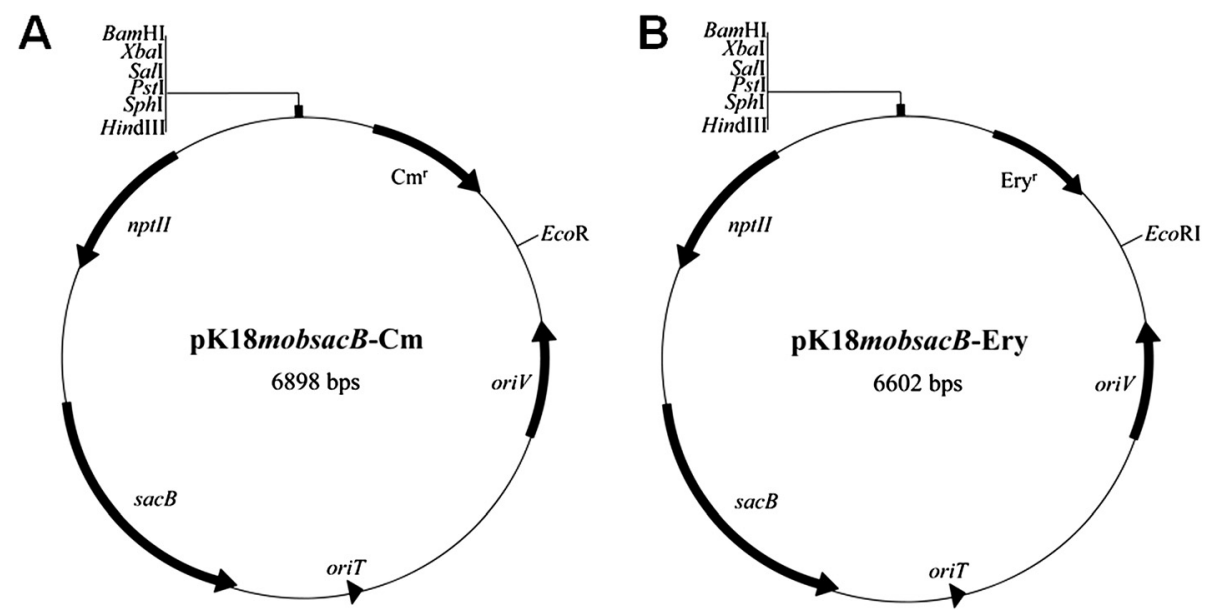

Figure 2 Maps of the pk18mobsacB-Cm (A) and pk18mobsacB-Ery (B) suicide vectors.

In-frame deletion of prodigiosin biosynthesis genes in DSM 6842

To test whether this system can be used in DSM 6842, pigM and pigK genes in an operon related to prodigiosin biosynthesis [26] were selected as a target region for deletion. A schematic illustrating the use of pK18mobsacB-Ery or pK18mobsacB-Cm as the knockout vector is shown in Figure 3. The pK18mobsacB-Ery plasmid was used to construct a pK18Ery-pigM-K suicide plasmid containing the homologous fragments upstream and downstream of the target region, and was then mobilized into DSM 6842 by conjugation (Figure 3A). After mating, cells were plated on $2216 \mathrm{E}$ containing erythromycin to screen for clones in which a single crossover event occurred. Two different crossover events occurred simultaneously: colonies $1,4,5$, and 6 were generated by $5^{\prime}$ recombination while colonies 2 and 3 were generated by $3^{\prime}$ recombination (Figure 4A and Figure 3B). Colony 1 from the first crossover was used for the second crossover. Sucrose counter-selection produced mutants without the pig $M-K$ region (Figure $3 C$ ). As shown in Figure 4B, deletion in colonies 1-3 was confirmed by PCR using primer set pigM-wS/pigM-wA, followed by DNA sequencing to further validate complete removal of the target region from the host genome. The 2025 bp region containing pigM-pigK genes was deleted in the $\Delta p i g M-K$ mutant. To check the phenotypic change of removing pig $M-K$ region in DSM 6842, prodigiosin production was monitored in the $\triangle p i g M-K$ strains and in the wild-type strain. As shown in Figure 4C, deletion mutants lost the ability to produce red pigment in $2216 \mathrm{E}$ medium. Thus conjugation-based genetic manipulation system is feasible for gene deletion in DSM 6842.

\section{In-frame deletion of bsmA in SM9913}

To test the feasibility of gene knockout in SM9913, bsmA, which encodes $E$. coli homolog biofilm stress and motility protein [27], was selected as a target gene. The pK18mob$s a c B-C m$ plasmid was used to construct a $\mathrm{pK} 18 \mathrm{Cm}-b s m A$ suicide plasmid containing the upstream and downstream regions of $b s m A$. Then $\mathrm{pK} 18 \mathrm{Cm}-b s m A$ was transformed into E. coli WM3064, and mobilized into SM9913 by conjugation. Three sets of primers were used to confirm plasmid integration into the host genome. As shown in Figure 5A, pK $18 \mathrm{Cm}-b s m A$ was integrated at the $3^{\prime}$ end in colonies $1-4$ to generate a $1078 \mathrm{bp}$ PCR product using primer set bsmA-dS/bsmA-wA, while the product length was 1510 bp for the wild-type SM9913. In addition, colonies 1-4 generated an extra 195 bp product using primer set bsmA-dS/bsmA-dA. Mutants from second homologous recombination were confirmed by PCR using primer set bsmA-wS/bsmA-wA. As shown in Figure 5B, after the removal of $b s m A$, PCR of $\triangle b s m A$ generated a 1919 bp product while the wild-type generated a 2351 bp product. To determine the effect of deleting $b s m A$ on SM9913, biofilm formation was measured in the $\triangle b s m A$ strain. In contrast to $b s m A$ in $E$. coli [27], deletion of $b s m A$ in SM9913 did not significantly alter biofilm formation under similar test conditions (Figure 5C). However, $\triangle b s m A$ formed a smaller swimming halo when compared to the wild-type strain, indicating that BsmA increases swimming motility in SM9913 (Figure 5D).

\section{In-frame deletion of $h m g A$ in SCSIO 04301}

To test the feasibility of gene knockout in SCSIO 04301, hmgA, encoding homogentisate 1, 2-dioxygenase [28], was selected as a target gene. As shown in Figure 6A, pK18Ery- $h m g A$ was integrated at the $3^{\prime}$ end in colony 1 to generate a 2636 bp PCR product using the hmgA-dS/ hmgA-wA primer set, while the product length was 3227 bp for the wild-type strain. As shown in Figure 6B, deletion in colony 1 was confirmed by PCR using the 


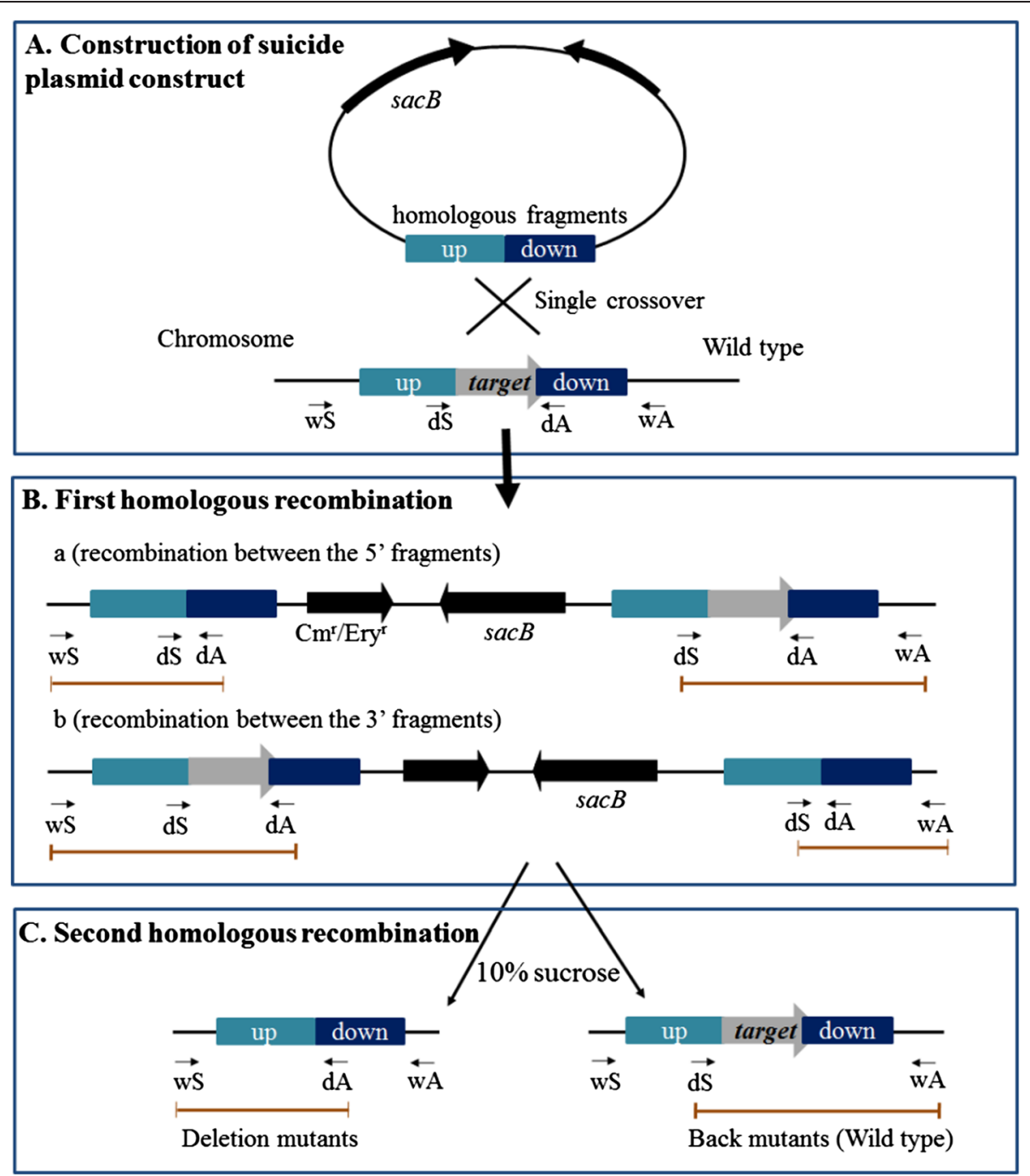

Figure 3 Schematics illustrating the use of the pK18mobsacB-Cm or pK18mobsacB-Ery suicide plasmids to generate a defined, marker-free deletion in Pseudoalteromonas strains. (A) Construction of suicide plasmid constructs containing the homologous fragments flanking the target DNA region. (B) First recombination event by integration into the chromosome via homologous recombination and position of the primers for plasmid insertion verification. (C) The second recombination event by sucrose selection, and position of primers to separate the deletion mutants from the wild-type.

hmgA-wS/hmgA-wA primer set, followed by DNA sequencing to further validate complete removal of the target region from the host genome. The 591 bp internal $h m g A$ region was deleted in the $\triangle h m g A$ mutant. To further confirm $h m g A$ gene deletion in SCSIO 04301, pigment production was checked in the $\triangle h m g A$ mutant strain. As shown in Figure 6C, as expected, inactivation of $h m g A$ leads to pyomelanin hyperproduction when cultured in $2216 \mathrm{E}$ medium for $48 \mathrm{~h}$. In addition, complementation of $h m g A$ via pWD2-oriT- $h m g A$ to $\triangle h m g A$ mutant strain rescued the wild-type phenotype, while the empty vector pWD2-oriT failed to do that (Figure 6C). Thus gene deletion and gene complementation is feasible in SCSIO 04301.
In-frame deletion of flagellar motor protein genes in SCSIO 11900

To test whether this system can be used in SCSIO 11900, fliF and fliG genes in an operon encoding flagellar motor proteins [28] were selected as a target region for deletion. As shown in Figure 7A, pK18Ery-fliFG was integrated at the 3 ' end in colony 1 to generate a 1698 bp PCR product using the fliFG-dS/fliFG-wA primer set, while the product length was $3414 \mathrm{bp}$ for the wild-type strain. As shown in Figure $7 \mathrm{~B}$, deletion in colony 1 was confirmed by PCR using the primer set fliFG-wS/fliFG-wA. The $1716 \mathrm{bp}$ region containing fliFG genes was deleted in the $\triangle$ fliFG mutant. To further confirm fliFG gene deletion in SCSIO 11900 , motility was measured in the $\triangle$ fliFG mutant strain. 
A

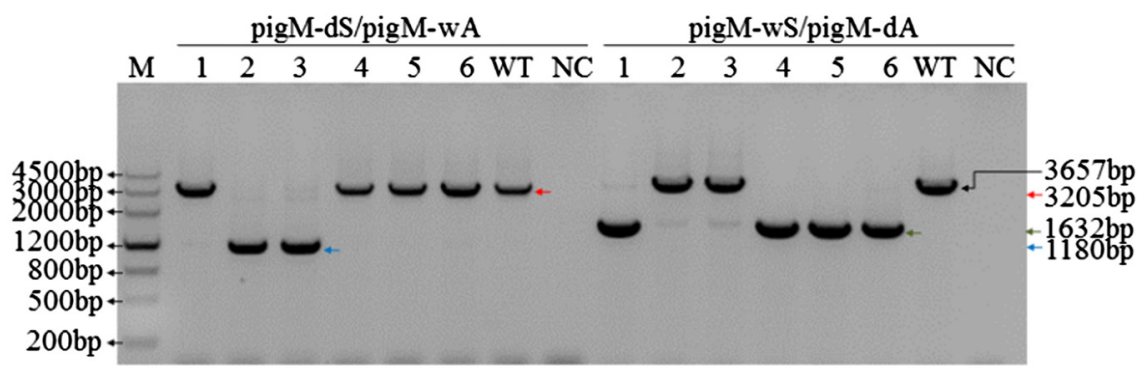

B

pigM-wS/pigM-wA C

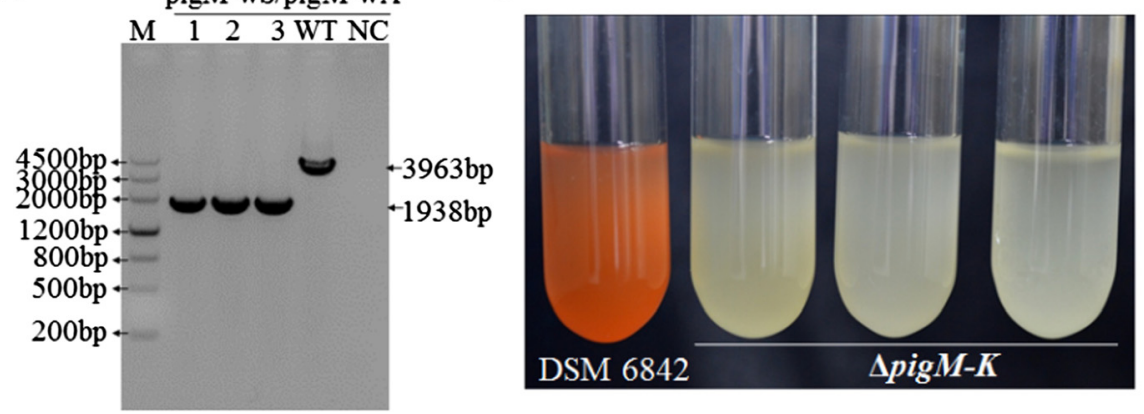

Figure 4 Confirmation of in-frame deletion of prodigiosin biosynthesis genes in DSM 6842. (A) PCR detection of pigM-K single crossover mutants using two primer pairs. M, DNA Marker III. 1-6, six independent colonies that can grow on the selective plate after conjugation. WT, DSM 6842 wild-type strain. $\mathrm{NC}$, negative control $\mathrm{dd}_{2} \mathrm{O}$. (B) PCR confirmation of the mutants that underwent a second homologous recombination using the pigM-wS and pigM-wA primers. 1-3, three independent colonies after the second homologous recombination. WT, wild-type strain DSM 6842. NC, negative control $\mathrm{ddH}_{2} \mathrm{O}$. (C) The prodigiosin production of three $\Delta$ pigM-K mutants and the wild-type strain DSM 6842.

A

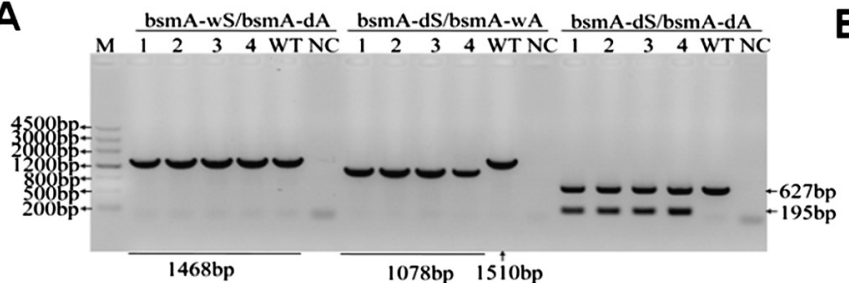

bsmA-wS/bsmA-dA bsmA-dS/bsmA-wA bsmA-dS/bsmA-dA

C

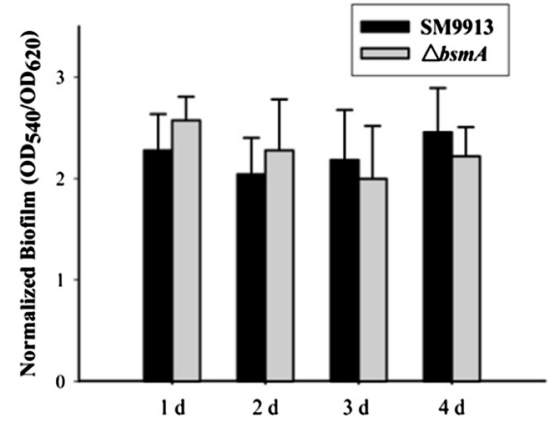

B

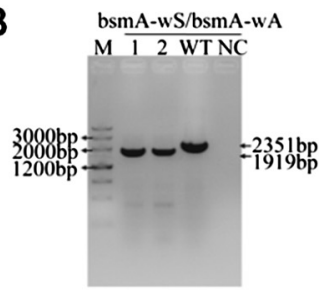

D

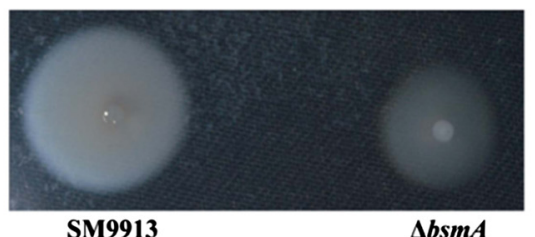

SM9913

Figure 5 Confirmation of in-frame deletion of bsmA in SM9913. (A) PCR detection of bsmA single crossover mutants using three primer pairs. M, DNA Marker III. PCR templates used are: 1-4, four independent colonies that can grow on the selective plate after mating. WT indicates SM9913 wild-type strain. NC indicates negative control dd $\mathrm{H}_{2} \mathrm{O}$. (B) PCR confirmation of the mutants that underwent a second homologous recombination using the bsmA-wS and bsmA-wA primers. 1-2, two independent colonies after the second homologous recombination. WT indicates SM9913 wild type. NC indicates negative control dd $\mathrm{H}_{2} \mathrm{O}$. (C) Biofilm formation of wild type SM9913 and $\Delta b s m A$ strains. Normalized biofilm formation (total biofilm/growth) in SWLB medium at $20^{\circ} \mathrm{C}$ after $1 \mathrm{~d}, 2 \mathrm{~d}, 3 \mathrm{~d}$, and $4 \mathrm{~d}$ in 96 -well plates. Data are the average of ten replicate wells from two independent cultures, and one standard deviation is shown. (D) Swimming motility of wild type SM9913 and $\Delta b s m A$. 


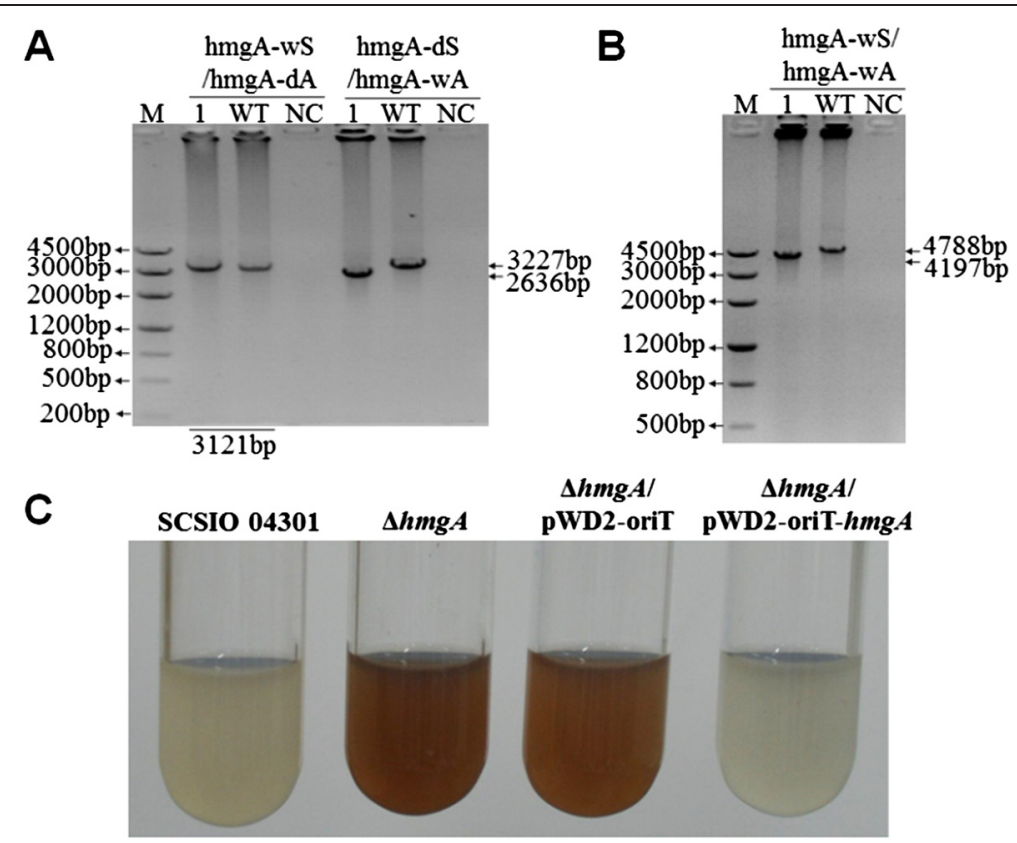

Figure 6 Confirmation of in-frame deletion of $h m g A$ in SCSIO 04301. (A) PCR detection of hmgA single crossover mutants using two primer pairs. M, DNA Marker III. PCR templates used are: 1, one colony that can grow on the selective plate after mating. WT indicates SCSIO 04301 wild-type strain. $\mathrm{NC}$ indicates negative control $\mathrm{ddH}_{2} \mathrm{O}$. (B) PCR confirmation of the mutants that underwent a second homologous recombination using the hmgA-wS and hmgA-wA primers. 1, one colony after the second homologous recombination. WT indicates SCSIO 04301 wild type. $\mathrm{NC}$ indicates negative control $\mathrm{ddH}_{2} \mathrm{O}$. (C) The pigment production of wild-type SCSIO 04301, $\Delta h m g A$ mutant, $\Delta h m g A / p W D 2-o r i T$ and $\Delta h m g A$ pWD2-oriT-hmgA.

As shown in Figure 7C, $\triangle$ fliFG mutant strain completely lost the ability of swimming motility compared with the wild-type strain. Thus conjugation-based genetic manipulation system is feasible for gene deletion in SCSIO 11900.

Different strain-specific genetic manipulation systems have been developed for Pseudoalteromonas strains TAC125 and SM9913 [14,15,29]. Here, we developed an efficient and generalizable genetic manipulation system for different Pseudoalteromonas strains. Incubation at $4^{\circ} \mathrm{C}$ has previously been used to select transconjugants and avoid overgrowth of the donor cells in the method developed for TAC125 $[14,29]$. However, conjugation at $4{ }^{\circ} \mathrm{C}$ is unfeasible for knockout of genes essential for cold tolerance or adaptation [15], and many Pseudoalteromonas strains grow too slowly at $4^{\circ} \mathrm{C}$. The mating temperature used in our approach was determined by the optimal growth temperature of the recipient strains, ranged from $20^{\circ} \mathrm{C}$ to $30^{\circ} \mathrm{C}$, overcoming the limitations of cold tempe rature conditions. E. coli ET12567 (pUZ8002) containing a suicide plasmid was used as the donor strain for gene deletion in SM9913, however, control experiments showed that the donor cells alone can still grow on the selection plate due to lack of donor cell-specific selection pressure [15]. In this study, the auxotrophic E. coli WM3064 strain was used as donor which cannot grow without the addition of DAP, thus can eliminate interference from donor cells and reduce false positive rates.
Most Pseudoalteromonas strains are sensitive to chloramphenicol and erythromycin, including all the strains tested in this study. When used chloramphenicol in the selection of mutants from the the single crossover event, a few false positive colonies may appear, however, this can be easily avoided by using erythromycin for the same purpose. The precise mechanism remains unclear, but it might be attributed to differences in antibiotic killing mechanisms. Therefore, erythromycin selective marker vector is recommended over chloramphenicol for Pseudoalteromonas genetic manipulations.

\section{Conclusions}

Here, we developed an efficient and generalizable genetic manipulation system for Pseudoalteromonas strains. Nine Pseudoalteromonas strains were sensitive to chloramphenicol and erythromycin, and thus both resistance genes were used to construct gene expression and gene knockout vectors. A conjugation transfer system was developed by modifying the culture conditions, which resulted in successful transfer of the pWD2-oriT gene expression vector from E. coli into Pseudoalteromonas strains with relatively high efficiency. By modifying a widely-used suicide plasmid (pK18mobsacB), a gene knockout system was developed and knockout of target DNA regions was confirmed in DSM 6842, SM9913, SCSIO 04301 and SCSIO 11900, covering from high or 


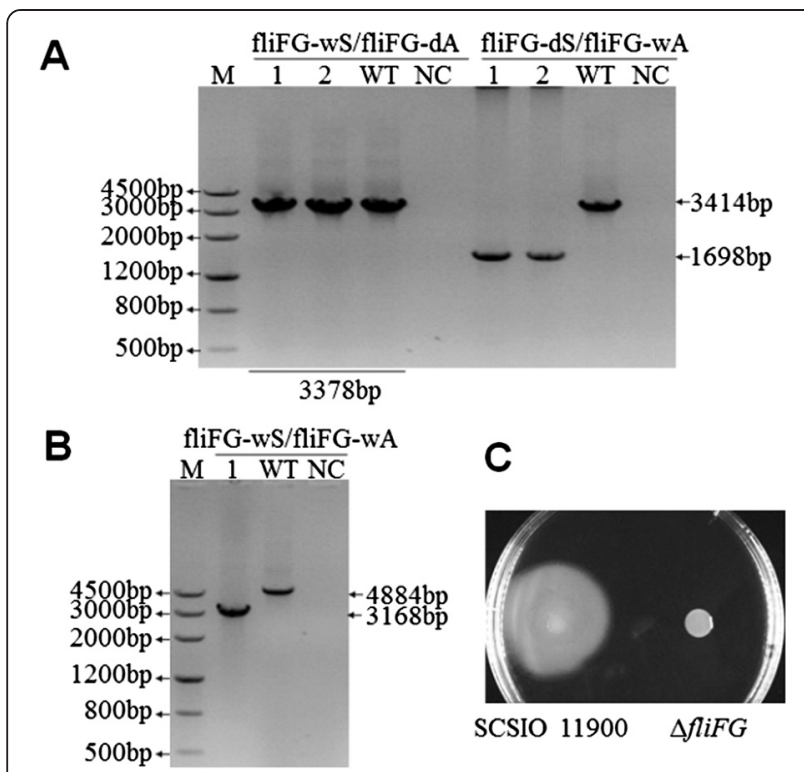

Figure 7 Confirmation of in-frame deletion of flagellar motor protein genes in SCSIO 11900. (A) PCR detection of fliFG single crossover mutants using two primer pairs. M, DNA Marker III. PCR templates used are: 1 and 2, two independent colonies that can grow on the selective plate after mating. WT indicates wild-type SCSIO 11900. NC indicates negative control $\mathrm{dd}_{2} \mathrm{O}$. (B) PCR confirmation of the mutants that underwent a second homologous recombination using the fliFG-wS and fliFG-wA primers. 1, one colony after the second homologous recombination. WT indicates wild-type SCSIO 11900. NC indicates negative control $\mathrm{ddH}_{2} \mathrm{O}$. (C) Swimming motility of wild-type SCSIO 11900 and $\Delta$ fliFG.

low conjugation efficiencies for pWD2-oriT transfer. In addition, gene complementation was also confirmed using this system. Taken together, conjugation-based genetic manipulation can be used efficiently for gene expression and gene deletion in Pseudoalteromonas strains, which will facilitate future in vivo studies of Pseudoalteromonas.

\section{Materials and methods}

Bacterial strains, plasmids, and growth conditions

The Pseudoalteromonas and E. coli strains and the plasmids used in this study are listed in Table 1. E. coli were cultured at $37^{\circ} \mathrm{C}$ in Luria-Bertani medium (LB) unless specified, and experiments with Pseudoalteromonas were conducted at $20-30^{\circ} \mathrm{C}$ in $2216 \mathrm{E}$ medium (BD Difco) or in SWLB medium (10 g peptone, and $5 \mathrm{~g}$ yeast extract dissolved in 11 artificial seawater). DAP (diaminopimelic acid) was added at $0.3 \mathrm{mM}$ to culture E. coli WM3064 strain. Conjugation assays were performed in modified LB mating medium (10 g peptone, $5 \mathrm{~g}$ yeast extract, $500 \mathrm{ml}$ artificial seawater, and $500 \mathrm{ml}$ distilled water) with $0.3 \mathrm{mM}$ DAP. When needed, antibiotics were added at the following concentrations: $100 \mu \mathrm{g} / \mathrm{ml}$ for ampicillin (Amp), apramycin (Apr) and spectinomycin (Spc); $50 \mu \mathrm{g} / \mathrm{ml}$ for kanamycin (Kan); $30 \mu \mathrm{g} / \mathrm{ml}$ for chloramphenicol $(\mathrm{Cm})$;
$25 \mu \mathrm{g} / \mathrm{ml}$ for erythromycin (Ery); and $10 \mu \mathrm{g} / \mathrm{ml}$ for gentamycin $(\mathrm{Gm})$ and tetracycline (Tet).

\section{Antibiotic sensitivity assay}

Sensitivities of Pseudoalteromonas strains to eight antibiotics were tested (Additional file 1: Table S1). Strains were grown in $2216 \mathrm{E}$ medium at $20-30^{\circ} \mathrm{C}$ to late exponential phase and then diluted to $10^{-2}-10^{-7}$ using 10 -fold serial dilutions prior to plating on $2216 \mathrm{E}$ containing each antibiotic. The plates were incubated at $20-30^{\circ} \mathrm{C}$ for $48 \mathrm{~h}$. Assays were performed in triplicate, and plates with no antibiotic were used as controls.

\section{Construction of pWD2-oriT and pWD2Ery-oriT mobilizable shuttle vectors}

The mobilization module containing a mob gene and the corresponding oriT region was amplified from pBBR1MCS2 using the oriT-F/oriT-R primer pair (Additional file 1: Table S2). The $1.5 \mathrm{~kb}$ PCR product was digested with $\mathrm{BamHI}$ and inserted into the corresponding site of pWD2 [9], resulting in the mobilizable shuttle vector pWD2-oriT. To generate the pWD2Ery shuttle vector, the Ery resistance gene was amplified from pHT304 [30] using the Ery-F/Ery$\mathrm{R}$ primer pair (Additional file 1: Table S2), and the $914 \mathrm{bp}$ PCR product was digested with $B a m H I / E c o R I$ and inserted into the corresponding sites of pWD2 [9]. To generate the pWD2Ery-oriT mobilizable shuttle vector, the $1.5 \mathrm{~kb}$ DNA region containing the mobilization module was derived from pWD2-oriT by digesting with $\mathrm{BamHI}$ and inserted into the corresponding site of pWD2Ery.

\section{Conjugation assays}

Conjugation experiments were performed as previously described [31] with some modifications. In brief, donor and recipient strains were grown to an $\mathrm{OD}_{600}$ of $0.8-1$, and $2 \mathrm{ml}$ donor cells and $1 \mathrm{ml}$ recipient cells were harvested by centrifugation (4000 rpm for $3 \mathrm{~min}$ ). Cells were washed twice with mating medium (MLB) and re-suspended in $100 \mu \mathrm{l}$ MLB containing DAP. The donor and recipient cells were mixed briefly, the mixture dropped on MLB with DAP plates, and the plates were incubated for $8 \mathrm{~h}$ or more until a lawn was formed at $20-30^{\circ} \mathrm{C}$. Cells were collected from the lawn and re-suspended in $2 \mathrm{ml}$ 2216E medium and spread onto 2216E plates with appropriate antibiotics to select the transconjugants. Transfer efficiency was calculated as the ratio of transconjugants per recipient cells for each condition.

\section{PCR and RAPD-PCR analysis}

Genomic DNA for PCR and random amplified polymorphic DNA (RAPD) analyses was isolated using a TIANamp Bacterial DNA Kit (Tiangen, Beijing, China). PCR primers are listed in Additional file 1: Table S2. Primers pWD2-S and pWD2-A were designed to amplify the 
replication region for detecting the pWD2-oriT plasmid in donor strains and transconjugants. RAPD-PCR analysis was performed using the 272 random primer (5'-AGCGG GCCAA-3') [32] to identify donor strains, recipient strains, and transconjugants based on amplification profiles. The RAPD-PCR reaction was: $95^{\circ} \mathrm{C}$ for $5 \mathrm{~min}$ follo wed by 40 cycles of $95^{\circ} \mathrm{C}$ for $45 \mathrm{~s}, 36^{\circ} \mathrm{C}$ for $1 \mathrm{~min}$, and $72^{\circ} \mathrm{C}$ for $2 \mathrm{~min}$; and $72^{\circ} \mathrm{C}$ for an additional $10 \mathrm{~min}$. Routine DNA manipulations were carried out following standard methods [33].

\section{Construction of the pK18mobsacB-Cm and pK18mobsacB-Ery suicide vectors}

The $1.2 \mathrm{~kb}$ DNA region containing the $\mathrm{Cm}$ resistance gene was recovered from pWD2 with $B a m H I / E c o R I$ and cloned into the commonly used $\mathrm{pK} 18$ mobsacB vector for gene knockout [24], resulting in the pK18mobsacB-Cm suicide vector. The Ery resistance gene was amplified from pHT304 [30] using the Ery-F/Ery-R primer pair, and the $914 \mathrm{bp} \mathrm{PCR}$ product was digested with BamHI/ $E c o$ RI and cloned into pK18mobsacB to produce the pK18mobsacB-Ery suicide vector.

\section{Construction of the $\triangle p i g M-K$ mutant strain in DSM 6842}

The suicide plasmid used for deletion of a DNA region containing pigM-K genes was based on pK18mobsacBEry. The schematic is shown in Figure 3. Two primer pairs (pigM-up-S/pigM-up-A and pigM-down-S/pigM-down-A) were used to amplify the upstream and downstream DNA sequences of the target region from DSM 6842 genomic DNA. The 996 bp and 815 bp PCR fragments were digested with $X b a \mathrm{I} / E c o$ RI and EcoRI/HindIII, respectively, cloned into the XbaI/HindIIII sites of pK18mobsacB-Ery, and were transformed into E. coli WM3064. Suicide plasmid pK18Ery-pigM-K was mobilized from E. coli WM3064 into DSM 6842 by intergeneric conjugation. After mating, cells were spread on 2216E plates containing erythromycin $(25 \mu \mathrm{g} / \mathrm{ml})$ to screen for clones in which the suicide vector pK18Ery-pigM- $K$ had integrated into the DSM 6842 genome via a single crossover event. The mutants were then grown at $25^{\circ} \mathrm{C}$ with shaking in $2216 \mathrm{E}$ medium without any antibiotics for $8 \mathrm{~h}$. To select mutants in which the second recombination had occurred, the culture was diluted and spread on 2216E medium containing $10 \%$ sucrose and grown at $25^{\circ} \mathrm{C}$ for $24-36 \mathrm{~h}$. Single colonies were transferred onto $2216 \mathrm{E}$ and 2216E containing $25 \mu \mathrm{g} / \mathrm{ml}$ erythromycin plates simultaneously, and colonies sensitive to erythromycin $(25 \mu \mathrm{g} / \mathrm{ml})$ were collected and confirmed by PCR followed by DNA sequencing.

Three other knockout mutants, $\triangle b s m A$ in SM9913, $\triangle h m g A$ in SCSIO 04301 and $\triangle f l i F G$ in SCSIO 11900, were constructed using the similar steps as $\triangle p i g M-K$, and detailed procedures can be found in the Additional file 1. The procedure for the complementation of $\Delta h m g A$ can also be found in the Additional file 1.

\section{Crystal violet biofilm and motility assays}

Biofilm formation was assayed in 96-well polystyrene plates (Corning Costar, Cambridge, MA) in SWLB medium at $20^{\circ} \mathrm{C}$ after $1 \mathrm{~d}, 2 \mathrm{~d}, 3 \mathrm{~d}$, and $4 \mathrm{~d}$ with crystal violet staining [34]. To account for growth effects, biofilm formation was normalized by dividing the total biofilm by the maximal bacterial growth as measured by turbidity at $620 \mathrm{~nm}$ for each strain. Cell motility was examined with $1 \%$ tryptone and $0.3 \%$ agar dissolved in seawater medium. Motility halos were quantified after $16 \mathrm{~h}$ using at least three plates for each condition and two independent cultures for each strain.

\section{Additional file}

\begin{abstract}
Additional file 1: Table S1. Antibiotic resistance of Pseudoalteromonas strains. Table S2. Primers used in this study. Figure S1. Growth test of the E. coli WM3064 donor strain and four recipient strains (DSM 16099, DSM 16098, SM9913, and DSM 6842) in LB with or without DAP, modified LB medium (MLB) with DAP, and 2216E with or without DAP. Figure S2. Confirmation of transconjugants by PCR. (A) PCR amplification with the pWD2-S/pWD2-A primer pair to detect pWD2-oriT in the transconjugants. (B) RAPD-PCR to confirm the host strain of the transconjugants. The templates in (A) and (B) are: Lane 1, donor strain E. coli WM3064/pWD2-oriT; Lane 2, SM9913; Lane 3-4, SM9913/pWD2-oriT; Lane 5, A37-1-2; Lane 6-7, A37-1-2/pWD2-oriT; Lane 8, DSM 16099; Lane 9-10, DSM 16099/pWD2-oriT; Lane 11, DSM 16098; Lane 12-13, DSM 16098/pWD2-oriT; Lane 14, SM20429; Lane 15-16, SM20429/pWD2-oriT; Lane 17, TAC125; Lane 18-19, TAC125/pWD2-oriT; Lane 20, DSM 6842; Lane 21-22, DSM 6842/pWD2-oriT. Lane 23, SCSIO 04301; Lane 24-25, SCSIO 04301/pWD2-oriT; Lane 26, SCSIO 11900; Lane 27-28, SCSIO 11900/ pWD2-oriT; M1, DL2K marker; M2, DNA Marker III.
\end{abstract}

Competing interests

The authors declare that they have no competing interests.

\section{Authors' contributions}

PW and XW designed the study, analyzed the data and wrote the manuscript. PW, BL, XC, ZZ and ZY participated in the experiments, and XC critically reviewed the manuscript. All authors read and approved the final manuscript.

\section{Acknowledgements}

This work was supported by the National Basic Research Program of China (2013CB955701), the National Science Foundation of China (31290233, 41406189 and 41230962 ) and the Chinese Academy of Sciences (XDA11030402). XW is the 1000-Youth Elite Program recipient in China.

\section{Author details}

${ }^{1}$ Key Laboratory of Tropical Marine Bio-resources and Ecology, Guangdong Key Laboratory of Marine Materia Medica, RNAM Center for Marine Microbiology, the South China Sea Institute of Oceanology, Chinese Academy of Sciences, Guangzhou 510301, China. State Key Laboratory of Microbial Technology, Marine Biotechnology Research Center, Shandong University, Jinan 250100, China. ${ }^{3}$ University of Chinese Academy of Sciences, Beijing 100049, China.

Received: 28 October 2014 Accepted: 10 January 2015

Published online: 23 January 2015

\section{References}

1. Gauthier G, Gauthier M, Christen R. Phylogenetic analysis of the genera Alteromonas, Shewanella, and Moritella using genes coding for small-subunit rRNA sequences and division of the genus Alteromonas into two genera, 
Alteromonas (emended) and Pseudoalteromonas gen. nov., and proposal of twelve new species combinations. Int J Syst Bacteriol. 1995;45:755-61.

2. Bian F, Xie BB, Qin QL, Shu YL, Zhang XY, Yu Y, et al. Genome sequences of six Pseudoalteromonas strains isolated from Arctic sea ice. J Bacteriol. 2012;194:908-9.

3. Ivanova EP, Sawabe T, Lysenko AM, Gorshkova NM, Svetashev VI, Nicolau DV, et al. Pseudoalteromonas ruthenica sp. nov., isolated from marine invertebrates. Int J Syst Evol Microbiol. 2002;52:235-40.

4. Kobayashi T, Imada C, Hiraishi A, Tsujibo H, Miyamoto K, Inamori Y, et al. Pseudoalteromonas sagamiensis sp. nov., a marine bacterium that produces protease inhibitors. Int J Syst Evol Microbiol. 2003:53:1807-11.

5. Park HJ, Shin SC, Kim D. Draft genome sequence of Arctic marine bacterium Pseudoalteromonas issachenkonii PAMC 22718. J Bacteriol. 2012;194:4140.

6. Yu M, Tang K, Shi X, Zhang XH. Genome sequence of Pseudoalteromonas flavipulchra JG1, a marine antagonistic bacterium with abundant antimicrobial metabolites. J Bacteriol. 2012;194:3735.

7. Xie BB, Shu YL, Qin QL, Rong JC, Zhang XY, Chen XL, et al. Genome sequences of type strains of seven species of the marine bacterium Pseudoalteromonas. J Bacteriol. 2012;194:2746-7.

8. Qin QL, Li Y, Zhang YJ, Zhou ZM, Zhang WX, Chen XL, et al. Comparative genomics reveals a deep-sea sediment-adapted life style of Pseudoalteromonas sp. SM9913. The ISME Journal. 2011;5:274-84.

9. Zhao DL, Yu ZC, Li PY, Wu ZY, Chen XL, Shi M, et al. Characterization of a cryptic plasmid pSM429 and its application for heterologous expression in psychrophilic Pseudoalteromonas. Microbi Cell Fact. 2011;10:30

10. Liu SB, Chen XL, He HL, Zhang XY, Xie BB, Yu Y, et al. Structure and ecological roles of a novel exopolysaccharide from the Arctic sea ice bacterium Pseudoalteromonas sp. strain SM20310. Appl Environ Microbiol. 2013;79:224-30

11. Bowman JP. Bioactive compound synthetic capacity and ecological significance of marine bacterial genus Pseudoalteromonas. Mar Drugs. 2007:5:220-41.

12. Wang YK, Zhao GY, Li Y, Chen XL, Xie BB, Su HN, et al. Mechanistic insight into the function of the C-terminal PKD domain of the collagenolytic serine protease deseasin MCP-01 from deep sea Pseudoalteromonas sp. SM9913: binding of the PKD domain to collagen results in collagen swelling but does not unwind the collagen triple helix. J Biol Chem. 2010;285:14285-91.

13. Zhang $W X$, Xie BB, Chen XL, Dong S, Zhang XY, Zhou BC, et al. Domains III and $\mathrm{I}-2$ alpha, at the entrance of the binding cleft, play an important role in cold adaptation of the periplasmic dipeptide-binding protein (DppA) from the deep-sea psychrophilic bacterium Pseudoalteromonas sp Strain SM9913. Appl Environ Microbiol. 2010;76:4354-61.

14. Giuliani M, Parrilli E, Pezzella C, Rippa V, Duilio A, Marino G, et al. A novel strategy for the construction of genomic mutants of the Antarctic bacterium Pseudoalteromonas haloplanktis TAC125. Methods Mol Biol. 2012;824:219-33.

15. Yu ZC, Zhao DL, Ran LY, Mi ZH, Wu ZY, Pang X, et al. Development of a genetic system for the deep-sea psychrophilic bacterium Pseudoalteromonas sp. SM9913. Microbl Cell Fact. 2014;13:13.

16. Yu M, Tang K, Liu J, Shi X, Gulder TA, Zhang XH. Genome analysis of Pseudoalteromonas flavipulchra JG1 reveals various survival advantages in marine environment. BMC Genomics. 2013;14:707.

17. Webber $M A$, Piddock $\sqcup$. The importance of efflux pumps in bacterial antibiotic resistance. J Antimicrob Chemother. 2003;51:9-11.

18. Thomas CM, Nielsen KM. Mechanisms of, and barriers to, horizontal gene transfer between bacteria. Nat Rev Microbiol. 2005;3:711-21.

19. Smillie C, Garcillan-Barcia MP, Francia MV, Rocha EP, de la Cruz F. Mobility of plasmids. Microbiol Mol Biol Rev. 2010;74:434-52.

20. Cascales E, Christie PJ. Definition of a bacterial type IV secretion pathway for a DNA substrate. Science. 2004;304:1170-3.

21. Schroder G, Lanka E. The mating pair formation system of conjugative plasmids-A versatile secretion machinery for transfer of proteins and DNA. Plasmid. 2005:54:1-25.

22. Kovach ME, Elzer PH, Hill DS, Robertson GT, Farris MA, Roop 2nd RM, et al. Four new derivatives of the broad-host-range cloning vector pBBR1MCS, carrying different antibiotic-resistance cassettes. Gene. 1995;166:175-6.

23. Dehio C, Meyer M. Maintenance of broad-host-range incompatibility group $\mathrm{P}$ and group Q plasmids and transposition of Tn5 in Bartonella henselae following conjugal plasmid transfer from Escherichia coli. J Bacteriol. 1997:179:538-40
24. Schafer A, Tauch A, Jager W, Kalinowski J, Thierbach G, Puhler A. Small mobilizable multi-purpose cloning vectors derived from the Escherichia coli plasmids pK18 and pK19: selection of defined deletions in the chromosome of Corynebacterium glutamicum. Gene. 1994;145:69-73.

25. Pelicic V, Reyrat JM, Gicquel B. Expression of the Bacillus subtilis sacB gene confers sucrose sensitivity on mycobacteria. J Bacteriol. 1996;178:1197-9.

26. Xie BB, Shu YL, Qin QL, Rong JC, Zhang XY, Chen XL, et al. Genome sequence of the cycloprodigiosin-producing bacterial strain Pseudoalteromonas rubra ATCC 29570(T). J Bacteriol. 2012;194:1637-8.

27. Weber MM, French CL, Barnes MB, Siegele DA, McLean RJ. A previously uncharacterized gene, yjfO (bsmA), influences Escherichia coli biofilm formation and stress response. Microbiology. 2010;156:139-47.

28. Zeng Z, Dai S, Xie Y, Tian X, Li J, Wang X. Genome sequences of two Pseudoalteromonas strains isolated from the South china sea. Genome Announc. 2014;2(2):e00305-14. doi:10.1128/genomeA.00305-14.

29. Duilio A, Tutino ML, Marino G. Recombinant protein production in Antarctic Gram-negative bacteria. Methods Mol Biol. 2004:267:225-37.

30. Arantes O, Lereclus D. Construction of cloning vectors for Bacillus thuringiensis. Gene. 1991;108:115-9.

31. Dominguez W, O'Sullivan DJ. Developing an efficient and reproducible conjugation-based gene transfer system for bifidobacteria. Microbiology. 2013;159:328-38

32. Nazik H, Ongen B, Erturan Z, Salcioglu M. Genotype and antibiotic susceptibility patterns of Pseudomonas aeruginosa and Stenotrophomonas maltophilia isolated from cystic fibrosis patients. Jpn J Infect Dis. 2007;60:82-6.

33. Sambrook JF, Russell DW. Molecular cloning: a laboratory manual. 3rd ed. Cold Spring Harbor, NY: Cold Spring Harbor Laboratory Press; 2001.

34. Pratt LA, Kolter R. Genetic analysis of Escherichia coli biofilm formation: roles of flagella, motility, chemotaxis and Type I pili. Mol Microbiol. 1998;30:285-93.

35. Rathgeber C, Yurkova N, Stackebrandt E, Schumann P, Humphrey E, Beatty $J$, et al. Metalloid reducing bacteria isolated from deep ocean hydrothermal vents of the Juan de Fuca Ridge, Pseudoalteromonas telluritireducens sp. nov. and Pseudoalteromonas spiralis sp. nov. Curr Microbiol. 2006;53:449-56.

36. Gauthier MJ. Modification of bacterial respiration by a macromolecular polyanionic antibiotic produced by a marine Alteromonas. Antimicrob Agents Chemother. 1976;9:361-6.

37. Medigue C, Krin E, Pascal G, Barbe V, Bernsel A, Bertin PN, et al. Coping with cold: the genome of the versatile marine Antarctica bacterium Pseudoalteromonas haloplanktis TAC125. Genome Res. 2005;15:1325-35.

\section{Submit your next manuscript to BioMed Central and take full advantage of:}

- Convenient online submission

- Thorough peer review

- No space constraints or color figure charges

- Immediate publication on acceptance

- Inclusion in PubMed, CAS, Scopus and Google Scholar

- Research which is freely available for redistribution 